\title{
Pattern Recognition of Pitch Motion for A New High Speed Gliding-hydrofoil Craft and Its Method
}

\author{
Gao Shasha, Yang Songlin, Liu Man, Shi Yan, Cao Zhiwei
}

\begin{abstract}
In this paper, a series of still water free decay experiments were carried out on a gliding-hydrofoil craft. By establishing the mathematical model of pitch motion mode, and based on system identification theory and genetic algorithm, the system identification software was written in $\mathrm{C \#}$ language. (1) A mathematical model of 3 kinds of pitch motion was established. The pitch motion of three different drafts and different initial pitch angles were identified and calculated. The conclusion is that the fitting effect of the equation 1 is the best; (2) By calculating the angular velocity forecasting values for various operating conditions, the errors between the experimental values and the fitted values were analysed. It is found that the consistency of the forecast value and the test result is good, which proves the reliability of the system identification software; (3) Based on the identification results, the variation of each hydrodynamic torque coefficient with draft and initial pitch angle analysed. The analysis results and methods can provide a reference for sea-keeping research of such unmanned craft, and provide important technical support for subsequent optimization design of craft type.
\end{abstract}

Index Terms - gliding-hydrofoil craft; pitch motion mode; pitching attenuation test; pattern recognition

\section{INTRODUCTION}

In the course of craft navigation, due to the interference of sea waves, sea wind and other environmental factors, the craft will produce a total of 6 degrees of freedom, such as rolling, pitching, yawing, swaying, surging and heaving. For rolling motion, the purpose of anti-rolling can be achieved by means of stabilizer and other control methods. But for pitching motion, there is no effective way. Therefore, the study of pitch motion is an important research topic in the field of craft motion.

At present, researches on pitching motion are mainly carried out in two aspects. The first is the theoretical calculation.

Such as slicing theory and slender body theory, and so on, and the use of theoretical calculation, to calculate and predict the craft's swing motion in the wave; on the other hand, the motion law of craft's pitching motion in certain water environment is studied by means of experiment, such as model test and so on. Wang Zhidong and so on ${ }^{[1]}$ by using the software FLUENT of computational fluid dynamics, developed the coupled solution of the longitudinal motion prediction program of the gliding craft, and carried out the numerical simulation of the motion response of the three-dimensional gliding boat model in the uniform flow,

Gao Shasha, Jingsu University of Science and Technology, Zhenjiang, Jiangsu, China, 18851406089

Yang Songlin, Jingsu University of Science and Technology, Zhenjiang, Jiangsu, China, 13705281656

Liu Man, Jingsu University of Science and Technology, Zhenjiang, Jiangsu, China, 13655282301 which provides an effective method for the real-time numerical prediction of the motion response of the gliding craft. According to the characteristics of the motion of high speed boats in still water and waves, a new method for predicting the longitudinal motion of high speed craft is proposed by Dong Wencai and other ${ }^{[2]}$ of the Naval Engineering University. This method can predict the longitudinal motion of the wave in the medium and high speed However, this method is not suitable for predicting the longitudinal motion of high speed craft at low velocity. Liu Cheng ${ }^{[3]}$ proposed a support vector machine prediction model based on particle swarm optimization and grey model. The model has high prediction accuracy. In view of the grey characteristics of the craft's longitudinal motion system, Zhao Shuang and others ${ }^{[4]}$ studied the prediction problem of the nonlinear longitudinal motion of crafts in sea waves by using the topological prediction method and the GM $(1,1)$ model The study of the craft's longitudinal motion prediction using topological prediction model is reasonable and feasible. Winer and others ${ }^{[5]}$ used the method of statistical prediction to predict craft's pitch, and Triantafyllou ${ }^{[6]}$ used the Calman filter method to predict the craft's pitching.

For the theoretical study of the craft's longitudinal motion, although it can reach the purpose of the motion prediction, there are still many problems to be obtained by theoretical calculation only. The correctness and reliability of the results are not easily verified, and the results of the theoretical study are verified by experimental research.

We generally refer to the test methods, including full-scale test and model test. Full-scale test usually refers to using full-scale craft to carry out a test on a specific sea surface after the completion of a craft, and to obtain data or curves representing the characteristics of the craft's motion. This method is harsh to the environment and it's expensive. It is difficult for the average scientific research institutions or researchers to undertake such a cost. The practical feasibility is low. The craft model test refers to the method of making the corresponding craft model in accordance with a certain scale of shrinkage for a real ship, making the corresponding craft model in the standard pool to obtain the results of the craft test. This method is relatively less expensive and more feasible than the actual craft test. In general we compare the results of the theoretical calculation with the test results to verify each other. This method is used in this paper.

In this paper, the model of high speed gliding craft model was tested and analyzed. The system identification mathematical model and method ${ }^{[7]}$ based on genetic algorithm was established. The identification software was developed and the identification analysis of the pitch motion was carried out. By analyzing the identification results and test data, the feasibility of the model was verified, and the reliability of system identification was verified. 


\section{SCHEME FOR PItch TeSt OF CRAFT MOdEL}

An optimized gliding craft model is selected for the pitching test. Its principle dimensions are shown in table I.

Table I. The main parameters of the optimized unmanned craft

\begin{tabular}{llll}
\hline Parameter & Symbol & value & Unit \\
\hline Length & $L$ & 1.50 & $\mathrm{~m}$ \\
Width & $B$ & 0.46 & $\mathrm{~m}$ \\
Depth & $D$ & 0.18 & $\mathrm{~m}$ \\
Draft & $T$ & 0.11 & $\mathrm{~m}$ \\
Displacement & $\Delta$ & 34.55 & $\mathrm{~kg}$ \\
Speed & $V$ & 8.00 & $\mathrm{kn}$ \\
\hline
\end{tabular}

The experiment was carried out at the towing tank of Jiangsu University of Science and Technology. The towing tank is 100 meters long, 6 meters wide, and the largest depth of water is 2 meters. In this paper, the attenuation law of craft model was determined by measuring the variation of pitch angle in the free attenuation process of static water. Before the test, the weight of the craft and the position of the center of gravity were measured before-hand. The specific test process is as follows:

(1)We prepared for trial the instrument including test model with length of 1.5 meters, MTi-G inertial measuring instrument (six degrees of freedom motion of measurable craft), data transmission line and computer;

(2)The MTi-G inertial measurement unit was placed horizontally on the center of gravity of the craft's model and connected to the equipment with data lines;

(3)The craft model was placed in the center of the pool, and the craft model was adjusted by loading weights to make it at the state of zero trim;

(4)When the model was at the state of zero trim, the pitch angle of the craft model was read by MTi-G software. By adjusting the horizontal position of the MTi-G, the initial pitch angle of the software was guaranteed to be between 0.01 degrees;

(5)When craft model was stable, the external force was applied according to the angle marking symbol. While releasing the external force, the MTi-G measuring instrument was used to collect data and allow the craft model to freely pitching. When the model was stable again, the data acquisition was stopped;

(6)In the process of repeating (4) - (5), the initial angle of pitching was changed. The initial angle of pitching was five groups of designed draft, including 1, 2, 3, 4, 5 degrees. For each group, the initial pitch angle was tested for 2-3 times, and the best one was selected as the effective data;

(7)The design draft pitch test was carried out by adding or reducing ballast by 1.1 and 0.9 times, repeated steps (4) (6).

\section{SYSTEM IDENTIFICATION EQUATION AND MATHEMATICAL MODEL}

The definition of Zadeh ${ }^{[8]}$ is that identification is based on input and output data, and determines a model that is equivalent to the measured system from a given set of model classes. Generally speaking, there are three elements in identification: data, models and criteria. Identification is to select a model that best fits the data in a set of model classes according to a criterion.

\section{Righting Moment}

When the craft is pitching at a certain angle, the center of gravity and the center of buoyancy are not on the same vertical line, which produces a moment to return the craft to its original position. This is the righting moment. The initial stability formula is as follows:

$$
M(\psi)=-D H \psi
$$

Where, $\mathrm{D}$ is the displacement of the craft and $\mathrm{H}$ is the initial stability height.

2. Damping moment

Pitch damping is a function of angular velocity, which $i$ s generally expressed as:

$M(\dot{\psi})=-2 N \dot{\psi}-W|\dot{\psi}| \dot{\varphi}-\mathrm{x} \dot{\psi}^{3}$

Where, $\mathrm{N}, \mathrm{W}$ and $\mathrm{x}$ are pitching damping moment coefficients.

3. Inertia moment

There is angular acceleration in the course of rotation of crafts, and the moment of inertia must be generated. The inertia moment of the pitch is made up of two parts. This is the inertia moment and additional moment of inertia of the craft itself. Generally speaking, they are linearly related to angular acceleration:

$$
M(\ddot{\psi})=-\left(I_{\mathrm{yy}}+J_{\mathrm{yy}}\right) \ddot{\psi}=-I_{\mathrm{yy}} \ddot{\psi}
$$

According to the dynamic balance principle of the body, the equilibrium condition of the craft model is $\sum M=0$, and the equilibrium equation of the craft model in still water is:

$$
M(\ddot{\psi})+M(\dot{\psi})+M(\psi)=0
$$

Based on the above equilibrium equations, three equations of pitch motion have been established for system identification analysis, and the pitching equation of craft model has been found.

Equation 1:

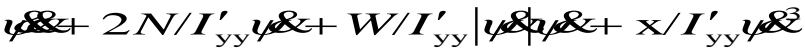

$$
\begin{aligned}
& +C_{1} / I_{\mathrm{yy}}^{\prime} \psi^{3}+C_{2} / I_{\mathrm{yy}}^{\prime} \psi^{2}+C_{3} / I_{\mathrm{yy}}^{\prime} \psi=\mathrm{O}
\end{aligned}
$$

(5)

Equation 2:

$$
\begin{aligned}
& \qquad \&+2 N / I_{\mathrm{yy}}^{\prime} \psi \&+W / I_{\mathrm{yy}}^{\prime} \mid \psi \& \alpha \& \&+\mathrm{x} / I_{\mathrm{yy}}^{\prime} \psi \&^{3} \\
& +D H / I_{\mathrm{yy}}^{\prime} \sin \psi=\mathrm{O}
\end{aligned}
$$

Equation 3:

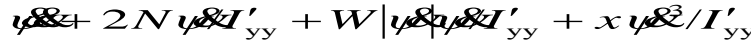

$$
\begin{aligned}
& +C_{1} \psi^{3} / I_{\mathrm{yy}}^{\prime}+C_{3} \psi / I_{\mathrm{yy}}^{\prime}=0
\end{aligned}
$$

Where, $h$ is initial stability height; $D$ represents the displacement; ${ }^{\prime}{ }^{\prime}{ }^{\prime}$ represents the total inertia moment; $\psi$ means the pitch angle; ${ }^{\psi} \&$ represents the angular velocity of pitch; represents the angular acceleration of the pitch; $2 N$ represents the coefficient of linear damping torque; $\mathrm{W}, \mathrm{X}$ representation of nonlinear damping moment coefficient; $C_{1}, C_{2}$ representation of nonlinear righting 
moment coefficient; ${ }^{C_{3}}$ represents the coefficient of linear righting moment.

Taking equation 2 as an example, we discretized it and got the following formula.

$$
\begin{aligned}
& \frac{\left(\psi \&_{K+1}-\psi \&_{K}\right)}{t}=-2 \frac{N_{1}}{I_{y y}^{\prime}} \psi \&_{K}-\frac{W_{1}}{I_{y y}^{\prime}}\left|\psi_{K}\right| \psi \&_{K} \\
& -\frac{\mathrm{x}_{1}}{I_{y y}^{\prime}} \psi \&_{K}^{3}-\frac{D H_{1}}{I_{y y}^{\prime}} \sin \psi_{K}
\end{aligned}
$$

The prediction of the pitch angular velocity at the $k+1$ moment is shown below.

$$
\begin{aligned}
& \psi_{K+1}^{X_{k}}=\left(-2 \frac{N_{1} t}{I_{y y}^{\prime}}+1\right) \psi \&_{K}-\frac{W_{1} t}{I_{y y}^{\prime}}\left|\psi \&_{K}\right| \&_{K} \\
& -\frac{\mathrm{x}_{1} t}{I_{y y}^{\prime}} \psi \&_{K}^{3}-\left(\frac{D H_{1} \sin \psi_{K}}{I_{y y}^{\prime}}\right) t
\end{aligned}
$$

By measuring the angle of the experimental $\mathrm{K}$, there are error estimation criteria at $\mathrm{K}+1$ time:

$$
E_{K+1}=\sum_{K+1}^{K}-\mathcal{R}_{K+1}
$$

According to the error estimation criterion, the objective function of pitch identification model is established as follows:

$$
F(x)=\sqrt{1 / N \sum_{k=1}^{N}\left(\psi_{K+1}^{K}-\psi_{K+1}\right)^{2}}
$$

The optimization mathematical model is composed of $\mathrm{d}$ esign variables, objective functions and constraints. In this ar ticle, the design variables include $I y \mathrm{y}, M 、 V 、 \mathrm{y}, \mathrm{h}$ 、 $C_{1} 、 C_{2} 、 C_{3}$. The constraint condition is the value range of each parameter to be identified. Based on experience, the range of parameters is shown below: $I y y \in[0,0.1$, $N \in[0,1], W \in[0,1], \mathrm{x} \in[0,10], \mathrm{H} \in[0,10]$, $C_{1} \in[0,10], C_{2} \in[0,10], C_{3} \in[0,10]$.

\section{SYSTEM IDENTIFICATION ANALYSES}

\section{A. Comparisons and Analyses of Experimental Data}

In the test process, each initial pitch angel tested 3 times , and the best data of the test data was selected as the effectiv e data. Due to human measurement factors, there was a certai $\mathrm{n}$ error in the test. The following figure I shows the initial pit ch angle is 5 degrees, and the draft is 0.9 times the draft of de sign draft, design draft and 1.1 times design draft.

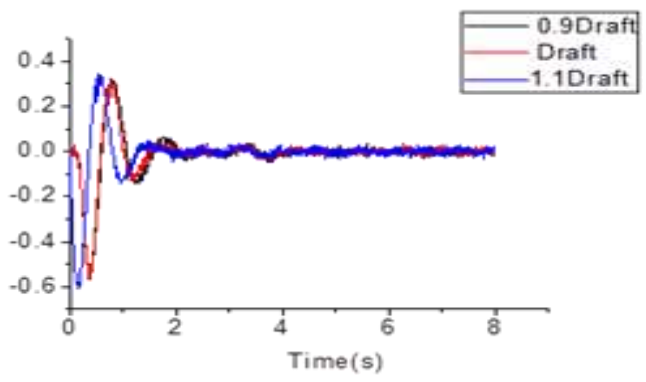

Fig. I Curves of angular velocity at initial angles of 5 degrees for different draft
As shown in figure I, when three different types of draft and initial pitch angle of 5 degrees, the peak value of the pitc $\mathrm{h}$ angular velocity increases and the pitch period decreases wi th the increase of the draft, which means that the ballast incre ases and the sea-keeping performance increases. When the cu rve is near 4 seconds, the attenuation law of the curve appear $\mathrm{s}$ anomalous state, which may be caused by the wave disturba nce caused by human factors and aggravate the pitch motion. It can be seen from the above diagram that the pitch velocity of the craft model is fast and the cycle is short, which is in lin e with the actual law.

\section{B. Analyses of Identification Results}

Genetic algorithm was selected to set the basic paramet ers as: optimization algebra was 2000, population size was 2 00 , the probability of variable carrier was $0.01-0.0001$, genet ic factor was 0.1 , and evolutionary weight was 0.9 .

The identification and calculation of three kinds of draft and different initial pitch angles were carried out with the id entification system of three different pitch mathematical mod els. The identification results are shown in table II.

Table II Identifying the value of the objective function of mathematical model

\begin{tabular}{lllll}
\hline $\begin{array}{l}\text { Objective } \\
\text { value }\end{array}$ & function & Equation 1 & $\begin{array}{l}\text { Equation } \\
2\end{array}$ & $\begin{array}{l}\text { Equation } \\
3\end{array}$ \\
\hline \multirow{2}{*}{$1^{\circ}$} & 0.9 & 0.012222 & 0.012278 & 0.012231 \\
& $\mathrm{~T}$ & 0.015295 & 0.015438 & 0.015298 \\
& 1.1 & 0.014992 & 0.015117 & 0.014997 \\
$2^{\circ}$ & 0.9 & 0.023923 & 0.024116 & 0.024039 \\
& $\mathrm{~T}$ & 0.019096 & 0.019205 & 0.019098 \\
& 1.1 & 0.021258 & 0.021507 & 0.021290 \\
$3^{\circ}$ & 0.9 & 0.025583 & 0.025673 & 0.025583 \\
& $\mathrm{~T}$ & 0.019560 & 0.019905 & 0.019565 \\
& 1.1 & 0.020014 & 0.020144 & 0.020014 \\
$4^{\circ}$ & 0.9 & 0.027312 & 0.027897 & 0.027589 \\
& $\mathrm{~T}$ & 0.018670 & 0.020233 & 0.018890 \\
& 1.1 & 0.023269 & 0.023592 & 0.023269 \\
$5^{\circ}$ & 0.9 & 0.015247 & 0.016047 & 0.015246 \\
& $\mathrm{~T}$ & 0.023616 & 0.024867 & 0.023734 \\
& 1.1 & 0.020237 & 0.020859 & 0.020238 \\
\hline
\end{tabular}

By comparing the objective function values of the above three equations, it is found that the objective function value of the 5 initial pitching angles and the 3 different drafts equat ion 1 is the least, indicating that the fitting effect of the equat ion 1 is the best. Taking the design of the angle of 3 degrees below the draft as an example, the identification equation is a s follows:

$$
\begin{aligned}
& 18.791293958 \psi \&+0.008501602 \mid \psi \& 4 \& \& \\
& +0.048391735 \psi^{3}+56.89821423 \psi^{3} \\
& +130.8204531 \psi^{2}+32.95335498 \psi=0
\end{aligned}
$$

In order to analyze the error of the test value and the fitting value, the experimental values of the angular velocity of designed draft and the initial angle of 3 degrees were compared with the fitting values identified by formula 1 , as shown in figure II. 


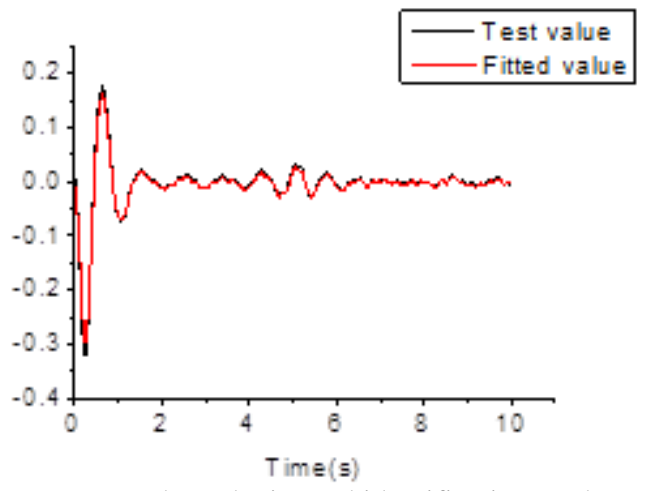

Fig. II Test angular velocity and identification angle velocity fitting curve

Table III Error table of experimental and identification results

\begin{tabular}{cccc}
\hline $\begin{array}{c}\text { Time } \\
(\mathrm{s})\end{array}$ & $\begin{array}{l}\text { Experimental } \\
\text { angular } \\
\text { velocity }\end{array}$ & $\begin{array}{l}\text { Identification } \\
\text { angular velocity }\end{array}$ & Error \\
\hline 0.60 & 0.124343 & 0.127320 & $2.33 \%$ \\
0.61 & 0.164479 & 0.161226 & $2.02 \%$ \\
0.62 & 0.143006 & 0.142625 & $0.27 \%$ \\
0.63 & 0.165123 & 0.168061 & $1.75 \%$ \\
0.64 & 0.170111 & 0.169394 & $0.42 \%$ \\
0.65 & 0.158781 & 0.159689 & $0.57 \%$ \\
0.66 & 0.134900 & 0.133365 & $1.15 \%$ \\
0.67 & 0.175247 & 0.176595 & $0.76 \%$ \\
0.68 & 0.158458 & 0.154440 & $2.60 \%$ \\
0.69 & 0.170776 & 0.169485 & $0.76 \%$ \\
0.70 & 0.130380 & 0.130956 & $0.44 \%$ \\
\hline
\end{tabular}

According to figure III, it can be seen that the fitting effect of test angular velocity and identification angular velocity is better. It can be seen from table 4.2 that the error between the test angle and the angular velocity is less than $3 \%$, which shows that the software is reliable and can predict the angular velocity at the next moment.

\section{Analyses of the hydrodynamic torque coefficient of the pitching}

Through the identification and calculation of 15 groups of pitching test data, the total inertia moment of the hull, the linear damping moment coefficient, the nonlinear damping moment coefficient and the linear righting moment coefficient and the nonlinear righting moment coefficient in each case were obtained. Among them, at different initial pitch angles, the hydrodynamic moment coefficient of the craft's pitch was measured with the draft shown in figure III-IX.

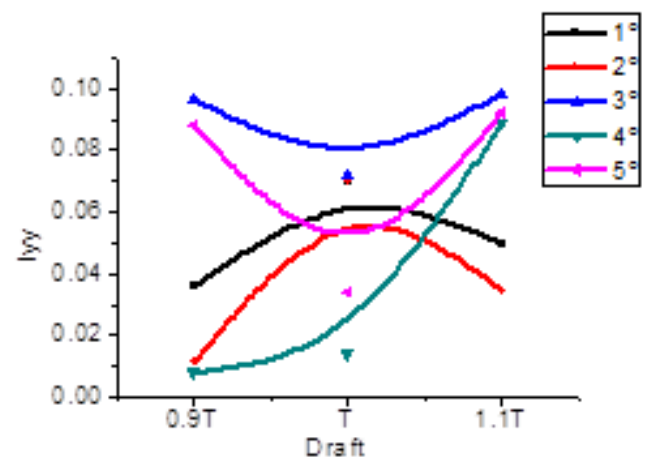

Fig. III The curves of the total moment of inertia $I_{\mathrm{yy}}$ of the pitch along with the initial pitch angle and draft

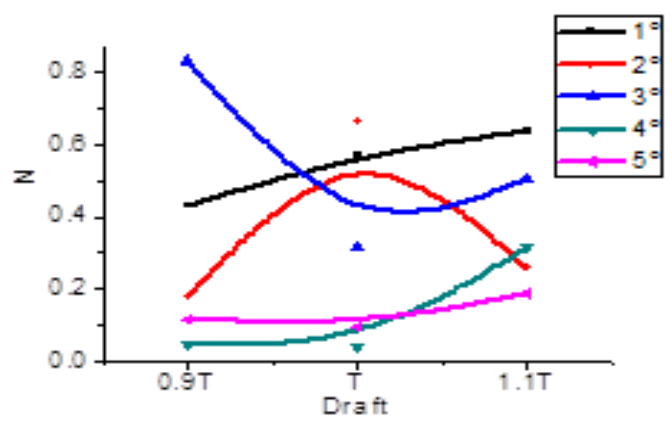

Fig. IV The curves of the linear damping coefficient $\mathrm{N}$ of the pitch along with the initial pitch angle and draft

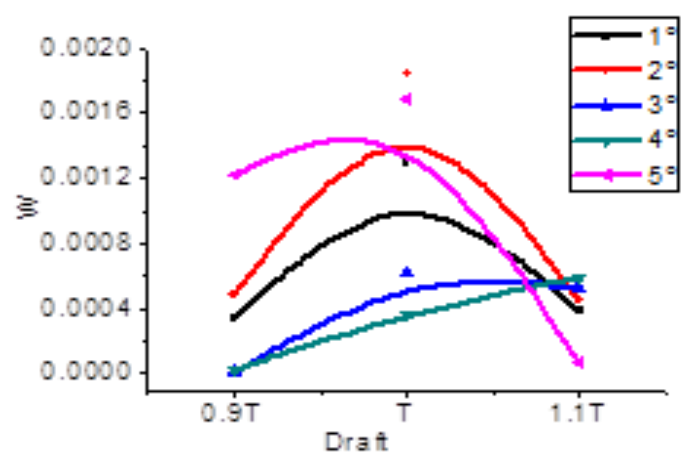

Fig. V The curves of damping moment coefficient $\mathrm{W}$ vary with initial pitch angle and draft

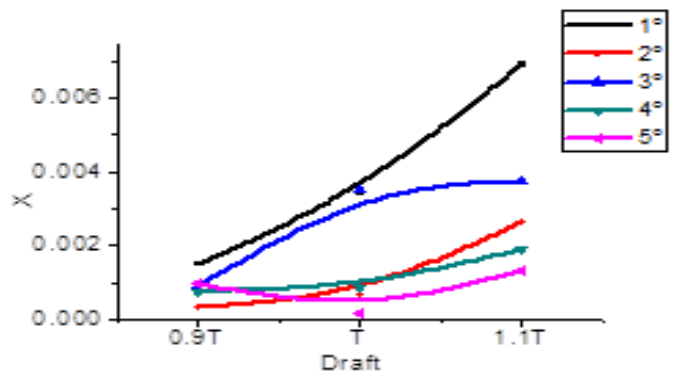

Fig. VI The curves of damping moment $\mathrm{X}$ vary with initial pitch angle and draft

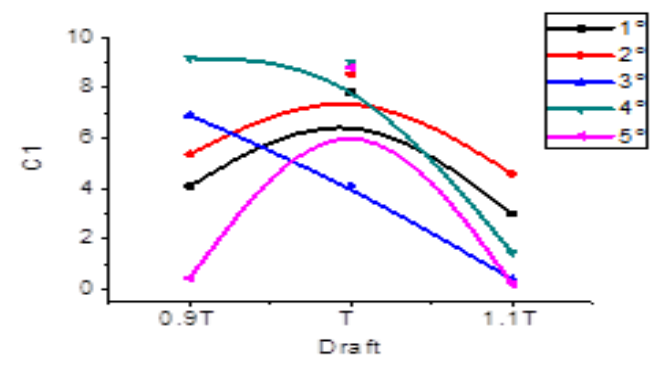

Fig. VII The curves of nonlinear righting moment coefficients $\mathrm{C} 1$ vary with initial pitch angle and draft

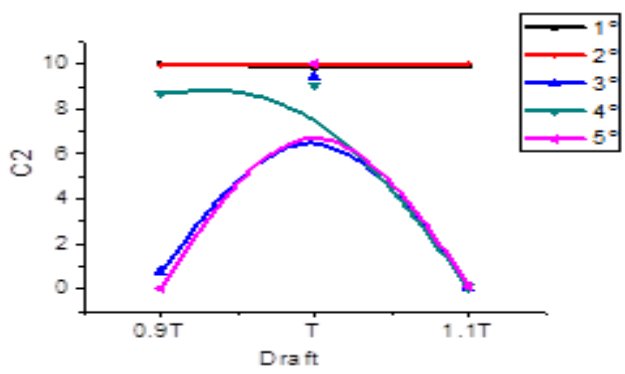

Fig. VIII The curves of nonlinear righting moment coefficients $\mathrm{C} 2$ vary with initial pitch angle and draft 


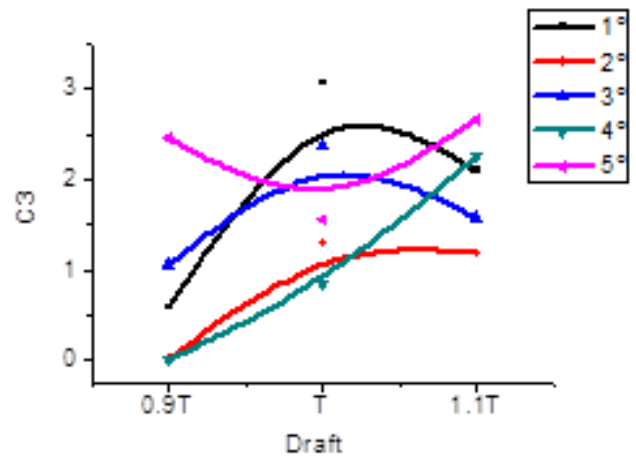

Fig. IX The curves of linear righting moment coefficient $\mathrm{C} 3$ with initial pitch angle and draft

It can be seen from figure IV-X that the values of the moment coefficients change with the change of draft and initial pitch angle. The total moment of inertia of the hull increases with the increase of displacement. The total moment of inertia at pitch angle 3 and 4 degrees decreases first and then increases. The linear damping moment coefficient was decreases with the increase of the initial pitch angle, but the linear damping moment coefficient was increases with the change of the draft. With the increase of initial pitch angle, the damping moment coefficient $\mathrm{W}$ varies little, and decreases with the increase of draft. The damping moment coefficient was increases with the increase of draft. With the increase of pitch angle, the variation of damping moment coefficient becomes smaller and smaller. The nonlinear righting moment coefficient $\mathrm{C} 1$ increases first and then decreases with the increase of draft. The nonlinear righting moment coefficient $\mathrm{C} 1$ is larger than that of draft at 3 degrees -5 degrees. The nonlinear righting moment coefficient $\mathrm{C} 2$ maintains a stable state with the change of draft at 1 degrees -2 degrees, and varies greatly with the change of draft at 3 degrees -5 degrees. The linear righting moment coefficient $\mathrm{C} 3$ increases with the increase of draft. To sum up, the nonlinear damping moment coefficient $\mathrm{W}$ and the nonlinear damping moment coefficient $x$ are smaller than the linear damping moment coefficient between 1 degree -5 degrees, indicating that the proportion of nonlinear damping is lower.

\section{CONCLUSION}

In this paper, the pitching motion mode of the gliding-h ydrofoil craft was studied through the still water pitching test and the system identification method. Through the identificat ion program, a mathematical model for static water pitch was found that is the most suitable for the craft model in 15 work ing conditions, which can accurately describe the process of $t$ he pitch motion and get the exact value of the moment coeffi cient. By analyzing the error of test angular velocity and iden tified angular velocity, the correctness of identification softw are was verified. Through system identification, the variation of each moment coefficient with the draft and angle of pitch was found. It shows that the system identification software ca $\mathrm{n}$ predict the future navigation parameters and movements of unmanned craft, and provide a reference for further research on the pitching motion of the gliding-hydrofoil craft.

\section{ACKNOWLEDGEMENT}

This project is funded by the following projects: The National Natural Science Foundation of China, the project approval number is 51379094 .

\section{REFERENCE}

[1] Wang Zhidong. Ling Hongjie. Real time numerical prediction method for heave and pitch motion of high speed planing craft [J]. Journal of Jiangsu University of Science and Technology (NATURAL SCIENCE EDITION), 2013, 27 (2): 113-118.

[2] Dong Wwncai. Wu Xiaoguang. A mathematical model for longitudinal motion of high speed craft in consideration of dynamic lift effect $[\mathrm{J}]$. Journal of Naval Engineering University, 2005, 7 (4):32-35.

[3] Liu Cheng. Li Keqi. Prediction model of craft pitch based on improved support vector machine $[\mathrm{J}]$. China Waterway Monthly, 2016(11):42-44.

[4] Zhao Shuang. An Yufang .Wang Huimin. Prediction of craft pitch motion based on topological prediction model [J]. Journal of Heilongjiang Institute of Technology, 2014, 28(1):71-74.

[5] Wiener N. Extrapolation, interpolation, and smoothing of stationary time series, with engineering appictions $[\mathrm{M}]$. Technology Press of the Massachusetts Institute of Technology; 1950.

[6] Triantafyllou M S, Bodson M B. Real time prediction of marine vessel motions [J], Using Kalman Filtering Techniques. 1982.

[7] She minghong. Analysis and research of genetic algorithm applied to system identification [J]. 2016, 11.

[8] Zadeh L A. Form circuit theory to system theory [J]. Proc. IRE, 1962, 59(5):856-865.

[9] Shao Kaiwen. Ma Yunyi. An introduction to craft technology and design [M].Bei Jing: National Defense Industry Press, 2005:44-58.

[10] Li Jide. Seakeeping [M].Harbin: Harbin Engineering University Press, 2007.

[11] Meng Fei. Xu Hongyang. Xie Zhibin. Parameter identification of craft pitch motion based on cloud particle swarm optimization algorithm $[\mathrm{J}]$ Craft Science and Technology, 2014, 36 (7):37-40.

[12] Yang Lili. Prediction of craft pitch parameters based on GM model [J] Foreign Electronic Measurement Technology, 2013, 32(3):55-57.

[13] Cui Ruonan. Reaearch on the parameter identification method of craft pitching and heaving motions based on improved particle swarm optimization [D]. Harbin Engineering University, 2015.

[14] Jin Wenju. Wang Zhidong. Li Lijun. Two dimensional numerical prediction of the heaving and pitching movement of a gliding boat $[\mathrm{J}]$. Research on Chinese craft, 2011, 6 (6):66-70.

[15] Dai Y, Liu L, Feng S. On the identification of coupled pitch and heave motions using opposition based particle swarm optimization [J]. Mathematical Problems in Engineering, 2014, 2014(3):1-10.

[16] Zhu Feng. Yang Songlin. Preliminary study of system identification method for composite trimaran's pitching and heave motion mode[C] China Conference on Control and Decision-making, 2014.

[17] Zhang Han. Ma Jianhong. Model analysis of pitch motion recognition based on genetic algorithm [J]. Craft Science and Technology, 2015, 37(11):35-38

[18] D Dumitru, Numerical investigation of a two degrees of freedom craft model for pitch - roll motion [C], IOP Conference Series: 10.1088/1757-899X/145/8/082007,2016.

[19] Deleanu D. Simultaneous resonance cases in a pitch - roll craft model PART 2: NUMERICAL ANALYSIS[C]// International Scientific Conference "engineering, Technology, Education, Security. 2016.

[20] Deleanu D. Comparative approximate studies on the craft's rolling motion [J]. 2015, 1:29-36.

[21] Hu A, Liu Y, Lu Y. Research on craft's nonlinear rolling motion based on multi-scale method [J]. Craft building of China, 2016.

Gao Shasha, Jingsu University of Science and Technology, Zhenjiang, Jiangsu, China, 18851406089

Yang Songlin, Jingsu University of Science and Technology, Zhenjiang, Jiangsu, China, 13705281656

Liu Man, Jingsu University of Science and Technology, Zhenjiang, Jiangsu, China, 13655282301. 